\title{
LA DIVERSITÉ FLORISTIQUE DES PELOUSES HUMIDES D'ALTITUDE: CAS DE QUELQUES SITES DU HAUT ATLAS MAROCAIN
}

\author{
Safia ALAOUI HARONI*, Mohamed ALIFRIQUI \& Ahmed OUHAMMOU \\ Laboratoire Ecologie Végétale et Environnement, Faculté des Sciences Semlalia, Université Cadi Ayyad, \\ BP 2390, Av My Abdellah Marrakech Maroc \\ *Auteur pour correspondance: alaoui_safia@yahoo.fr
}

Recibido el 10 de septiembre de 2009, aceptado para su publicación el 7 de octubre de 2009

Publicado "on line" en octubre de 2009

\begin{abstract}
RÉSUMÉ. La diversité floristique des pelouses humides d'Altitude: cas de quelques sites du Haut Atlas Marocain. Dans le Haut Atlas, les pelouses humides et pozzines constituent des pâturages assez convoités. Leur flore se caractérise par une grande richesse spécifique: 165 taxons inventoriés dans la zone d'étude (plateau de l'Oukaimeden, plateau de Tichka et l'amont de la haute vallée d'Ait Mizane) avec un taux d'endémisme estimé à 30,3\% et un degré de rareté qui atteint $31 \%$. Dans un contexte dominé par l'élément méditerranéen, la flore de ces pelouses est très enrichie de souches septentrionales $15,7 \%$, repoussées vers nos latitudes méridionales lors des dernières glaciations. Ces zones humides vulnérables de par leur structure, fonctionnement écologique et état de conservation, se trouvent très touchées par les changements climatiques et les mutations environnementales récentes.
\end{abstract}

Mots clé. Pelouses humides d'altitude, biodiversité floristique, Haut Atlas, Maroc.

SUMMARY. Floristic biodiversity of the High elevation wet grasslands: case of some Moroccan High Atlas sites. In the High Atlas mountains, the wet grasslands and pozzines constitute a highly coveted pastoral resource. Its flora is characterized by a great specific richness: 165 taxa are recorded in the study area (Oukaimeden plateau, Tichka plateau and the upstream of the Ait Mizane high valley) and schow a rate of endemism estimated at $30.3 \%$ and a degree of rarity reaching $31 \%$. In a Mediterranean context, the wet grasslands flora is supplemented with plants of northern origin $15.7 \%$, pushed towards the extreme southern latitudes during the last glaciations. These wet pastures, highly sensitive by their structure, ecological functions and their conservation state, are very affected by the climatic changes and the global environmental changes.

Key words. Wet grasslands, floristic biodiversity, High Atlas, Morocco.

\section{INTRODUCTION}

Dans les hautes altitudes du Haut Atlas marocain, la géomorphologie et l'évolution des versants encadrants a permis la prolifération de pelouses humides et de pozzines se développant au niveau des dépressions et replats où il $\mathrm{y} a$ accumulation de matériaux fins (sable, argile) 
et de matériel humique qui permettent une bonne rétention des eaux des précipitations et des fontes de neige (Dresch, 1941; Quézel, 1957; Gauquelin, 1988). Ce sont d'ailleurs les seuls groupements végétaux d'altitude où il est possible de reconnaître un sol, parfois complexe, mais souvent sans horizons discernables. Les originalités floristiques et édaphiques de ces zones font d'elles de très bons témoins de l'histoire botanique du Haut Atlas. Certaines pozzines ont fait l'objet d'études de palynologie (Reille, 1976), de pédoanthracologie (Thinon $\&$ Alifriqui, 2004) et de datation (El Graoui et al., 2008) permettant de reconstituer l'histoire botanique du Haut Atlas et à travers lui celle des autres systèmes montagneux nord africains.

Par leur richesse et leur productivité de biomasse fourragère et nutritive, les pelouses humides sont très convoitées par la population des vallées riveraines pour le pâturage. Elles subissent une gestion traditionnelle (Berque, 1955) dans un ensemble dominé par une exploitation pastorale séculaire. Les sites étudiés ont constitué depuis très longtemps des pâturages collectifs soumis à une gestion communautaire ancestrale « Agdal » (Ilahiane, 1990; Auclair \& Alifriqui, 2005; Mahdi 1999). Cette gestion particulière de l'espace et des ressources consiste à limiter les droits d'accès et/ou d'usages à la loi coutumière du groupe. La mise en défens temporaire respecte le fonctionnement écologique et phénologique et permet la reconstitution du milieu, la conservation de la biodiversité et la pérennité de la ressource (Alaoui Haroni \& Alifriqui, 2009).

Le présent travail consiste en une analyse quantitative et qualitative de la flore des pelouses humides des sites Ait Mizane, Oukaimeden et Tichka à travers des paramètres très significatifs: richesse spécifique (nombre de taxons), types biologiques, origine biogéographique, endémisme et rareté.

\section{Présentation de la zone d'étude}

Le premier site est le plateau de $1^{\prime}$ Oukaimeden $\left(7^{\circ} 52^{\prime}\right.$ x $\left.31^{\circ} 12^{\prime}, 2600 \mathrm{~m}\right)$ situé à $75 \mathrm{~km}$ au sud de Marrakech et s'élève à $2600 \mathrm{~m}$ d'altitude. Il fait partie du Haut Atlas siliceux avec substrat précambrien éruptif acide à rhyolites et andésites. Le bioclimat est de type méditerranéen subhumide à hiver très froid, l'enneigement est important entre décembre et mars. La pratique d'Agdal y est toujours respectée (la date de fermeture est le 15 mars et d'ouverture est le 10 août), avec une forte pression touristique.

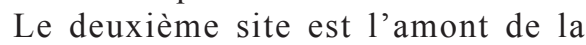
haute vallée d'Ait Mizane ( $7^{\circ} 56^{\prime}$ x $31^{\circ} 03^{\prime}$, $3000 \mathrm{~m}$ ), situé dans le grand bassin de Gheraya $70 \mathrm{~km}$ au sud de Marrakech. Il est dominé par des terrains précambriens et primaires à substrat éruptif acide. Le bioclimat est méditerranéen humide à hiver très froid; la période d'enneigement varie entre 4 et 5 mois. La pratique de l'Agdal y est en déclin, avec une forte pression pastorale et touristique.

Le troisième site est le plateau de Tichka $\left(8^{\circ} 34^{\prime} \mathrm{x} 31^{\circ} 55^{\prime}, 2700 \mathrm{~m}\right)$ situé à plus que $170 \mathrm{~km}$ au sud de Marrakech. Il fait partie du Haut N'Fis et dominé par des substrats granitiques. Le bioclimat est méditerranéen subhumide à humide à hiver très froid; la période d'enneigement dure 4 à 5 mois. La pratique d'Agdal y est bien conservée (la mise en défens débute avec les premières fontes des neiges jusqu'au 17 juillet) et une très faible fréquentation touristique.

\section{MATERIEL ET METHODE}

Des campagnes de terrain ont permis le choix des points d'échantillonnage et la récolte de la flore. 85 relevés floristiques (10 à Ait Mizane, 20 à l'Oukaimeden et 55 à Tichka) sont effectués sur l'aire minimale qui varie en fonction des faciès et des sites étudiés (Godron, 1971; Guinochet, 1973). La géomorphologie et le gradient d'humidité du sol permettent l'individualisation des faciès 


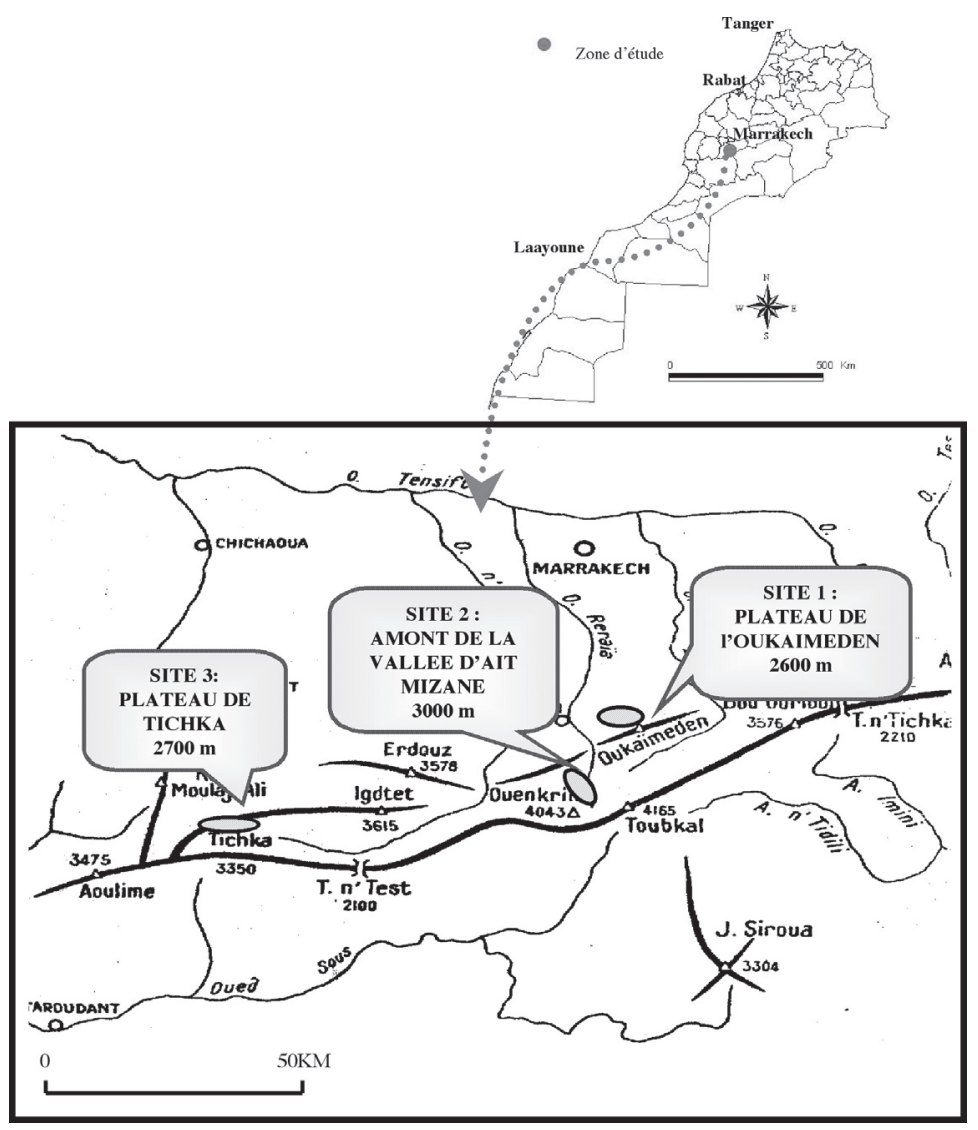

Figure 1: Situation géographique de la zone d'étude. Geographic situation of the study area.

de végétation à physionomie et à contenu floristique assez variés. Ainsi, on distingue:

- Le faciès très humide avec pozzines à Nardus stricta L. et Carex intricata Tineo

- Le faciès humide à Ranunculus aurasiacus Pomel

- Le faciès moyennement humide à Festuca maroccana Trabut \& St-Yves

Les relevés floristiques sont réalisés dans les pelouses hautes plus de $10 \mathrm{~cm}$; au deçà de cette hauteur des quadrats de $30 \mathrm{~cm}$ de côté sont prélevés et conservés (cas de certaines pelouses à Ait Mizane) au laboratoire dans des bassines régulièrement irriguées et à température ambiante afin de permettre l'expression du maximum de stock de graines dans le sol.

Les spécimens récoltés sont conservés à l'herbier de la Faculté des Sciences SemlaliaMarrakech et au Muséum d'Histoire Naturelle de Marrakech. Leur détermination a été établie à l'aide de la Flore pratique du Maroc (Fennane et al, 1999; 2007), de la Nouvelle flore de l'Algérie et des régions désertiques méridionales (Quézel et Santa 1962-1963) et sur la collection de l'herbier de la Faculté des Sciences Semlalia-Marrakech. La nomenclature utilisée correspond à celle du catalogue des plantes du Maroc (Jahandiez et Maire, 19311932-1934 et Emberger \& Maire, 1941) et celle de la Flore pratique du Maroc (Fennane et al. 
1999; 2007).

La détermination du statut des taxons endémiques, rares ou menacés est faite à partir du Catalogue des plantes vasculaires rares, menacées ou endémiques du Maroc (Fennane \& Ibn Tattou, 1998). Ce catalogue adopte quatre catégories d'endémisme: E: Endémique du Maroc; I: Endémique du Maroc et de la Péninsule Ibérique; A: Endémique du Maroc et de l'Algérie; IA: Endémique du Maroc, de la Péninsule Ibérique et de l'Algérie.

Et six catégories de rareté: $\mathrm{R}$ : Taxon rare, qui est en général signalé dans 1 ou 2 divisions du Catalogue des plantes du Maroc de Jahandiez \& Maire (1931-1934) et Emberger \& Maire (1941). RR: Taxon très rare; nombre de localités connues $\leq 5$. $\mathrm{R}$ ?: Taxon soupçonné rare. RR?: Taxon soupçonné très rare. ??: Taxon éteint ou de présence douteuse. V: Vulnérable (ou semble l'être); en voie de régression et pourrait devenir rare à court terme.

La détermination de l'origine biogéographique de la flore des pelouses humides dans les trois sites étudiés a été faite essentiellement sur la base des travaux de Galland (1988)*, complétée par le Catalogue des plantes vasculaires du Nord du Maroc (Valdés et al., 2002)**, La Nouvelle flore d'Algérie et des régions désertiques méridionales (Quézel et Santa 1962-1963)*** et du Manuel de la détermination de la flore valencienne (Mateo Sanz \& Crespo, 2001)**** et à partir du site www.tela-botanica.org

\section{RESULTATS}

\section{PTERYDOPHYTES OPHIOGLOSSACEAE}

\section{Botrychium lunaria L.}

RR, Pozzines, suintements et pelouses très humides, Europe, Asie tempérée, N Amérique, Patagonie, Australie, Tasmanie**.

Ait Mizane, Oukaimeden, Tichka.

\section{POLYPODIACEAE}

\section{Asplenium trichomanes L.}

Suintements et pelouses très humides, Circumboréal $* * *$.

Tichka.

Cystopteris fragilis (L.) Bernh.

Suintements et bords des ruisseaux, Subcosmopolite**.

Tichka.

\section{Polypodium vulgare L.}

RR, Suintements et pelouses très humides, Europe, Région Méditerranéenne**.

Tichka.

\section{ANGIOSPERMES DICOTYLEDONES APIACEAE}

Apium nodiflorum (L.) Koch.

Pelouses très humides, bords des ruisseaux, Atlantique, Eurasiatique-méditerranéen*. Oukaimeden, Tichka.

Apium repens Jacq.

Bords des ruisseaux et du lac, pelouses fortement pâturées, Européen-méditerranéen*. Oukaimeden.

Bunium alpinum Waldst. \& Kit. subsp. atlanticum (Maire)

E, pelouses humides et moyennement humides Oukaimeden, Tichka.

Carum atlanticum Litard. \& Maire

E, R, Pozzines.

Ait Mizane, Tichka.

\section{Conium maculatum $\mathrm{L}$}

Pelouses humides, prés des Azibs, Nord Afrique, Eurasie**.

Oukaimeden, Tichka.

Eryngium variifolium Coss.

E, Pozzines, pelouses très humides et bords des ruisseaux. Ait Mizane, Oukaimeden, Tichka.

Heracleum sphondylium (L.) Litard. subsp. embergeri Maire

$\mathrm{E}, \mathrm{R}$, Pelouses très humides, humides et bords des ruisseaux.

Oukaimeden, Tichka.

\section{ASTERACEAE}

Anacyclus pyrethrum $\mathrm{L}$. 
Pelouses moyennement humides, prés des Azibs et sentiers, Ibéro-maghrébin*.

Oukaimeden, Tichka.

Bellis caerulescens Coss.

E, Pozzines et pelouses très humides.

Ait Mizane, Oukaimeden, Tichka.

Cirsium chrysacanthum (Ball) Jahand.

$\mathrm{E}, \mathrm{R}$ ?, bords des suintements, pelouses très humides et humides.

Ait Mizane, Oukaimeden, Tichka.

\section{Crepis pulchra L.}

Pelouses très humides et humides, $\mathrm{C}$ et $\mathrm{S}$ Europe, N Afrique, W, C et SW Asie**.

Oukaimeden.

Erigeron mairei $\mathrm{Br}-\mathrm{Bl}$.

E, Pozzines.

Ait Mizane, Tichka.

Heliocauta atlantica Lit. et Maire

E, R ?, Pozzines.

Ait Mizane.

Hieracium pseudopillosella Ten.

Pelouses très humides et humides, Européenméditerranéen*.

Ait Mizane, Oukaimeden, Tichka

Hypochoeris arachnoidea Poir.

Pelouses moyennement humides, Maroc, Algérie**.

Oukaimeden, Tichka.

Jurinea humilis DC.

Pelouses moyennement humides et sèches, Ouest-méditerranéen***.

Ait Mizane, Oukaimeden, Tichka

Lactuca virosa L. subsp. cornigera (Pau\& Font

Quer) Emberger

$\mathrm{E}, \mathrm{R}$ ?, pelouses humides, moyennement

humides et pelouses sèches (rudérales)

Oukaimeden, Tichka.

Leontodon atlanticus (Ball.) Wimmer

E, Pozzines et pelouses très humides.

Ait Mizane, Oukaimeden, Tichka.

Mantisalca salmantica (L.) Briq. Et Cavill.

Pelouses moyennement humides et sèches, Méditerranéen**.

Ait Mizane, Oukaimeden, Tichka.

Senecio giganteus Desf.

Pelouses humides et moyennement humides, Maroc et N Algérie**.

Oukaimeden, Tichka.

Sonchus maritimus L. subsp. aquatilis (Pourr.) Nyman
Orient**.

Bords du lac, Région Méditerranéenne, Moyen

Oukaimeden.

Taraxacum sp.

Pelouses humides et moyennement humides, Méditerranéen****.

Ait Mizane, Oukaimeden, Tichka.

Tolpis barbata (L.) Gaertn. subsp. liouvillei (Br-Bl

\& Maire) H. Lindb. fil

E, Pelouses humides et moyennement humides.

Oukaimeden, Tichka.

\section{BORRAGINACEAE}

Cynoglossum watieri (Batt. \& Maire) Br-Bl. \& Maire

E, pelouses moyennement humides et sèches

prés des Azib (Rudérale).

Oukaimeden, Tichka.

Echium plantagineum $\mathrm{L}$.

Pelouses moyennement humides et sèches

(Rudérale), W et S Europe, N Afrique, SW Asie, Macaronésie (à l'exception de Cap Vert)**.

Oukaimeden.

Lithospermum arvense $\mathrm{L}$.

Pelouses moyennement humides, Méditerranéen***.

Tichka.

Myosotis atlantica Vestergr.

E, Pozzines, pelouses très humides et bords des ruisseaux.

Ait Mizane, Oukaimeden, Tichka.

\section{BRASSICACEA}

Arabis alpina L. subsp. caucasica (Willd.) Briq

Pelouses moyennement humides et sèches,

Méditerranéen*.

Tichka.

Arabis coringioides Ball.

E, Pozzines.

Ait Mizane, Tichka.

Erysimum nervosum Pomel

Pelouses moyennement humides et sèches,

NW Afrique*.

Tichka

Lepidium hirtum L. subsp. atlanticum (Ball)

Maire

E, Pozzines, suintements et bords du lac.

Ait Mizane, Oukaimeden, Tichka.

Nasturtium officinale R.Br. 
Suintements, sources et ruisseaux, Cosmopolite*.

Oukaimeden, Tichka.

Rorippa atlantica (Ball.) Maire

E, Pozzines, pelouses très humides et suintements.

Ait Mizane, Tichka.

\section{CAMPANULACEAE}

\section{Campanula filicaulis DR.}

Pozzines, pelouses très humides et humides, Maghrébin*.

Oukaimeden, Tichka.

Jasione sessiflora Boiss. \& Reuter

Pelouses moyennemnt humides et sèches, Méditerranéen*****.

Tichka.

\section{CARYOPHYLLACEAE}

Cerastium arvense subsp. arvense

RR ?, Pozzines, Boréo-montagnard*.

Ait Mizane, Oukaimeden, Tichka.

Cerastium cerastoides (L.) Britton

RR, Pozzines et bords des ruisseaux, Boréomontagnard*.

Ait Mizane, Oukaimeden, Tichka.

\section{Herniaria glabra L.}

Pelouses moyennement humides et sèches, Européen-méditerranéen*.

Ait Mizane, Oukaimeden, Tichka.

Minuartia tenuissima (Pomel) Mattf.

Pelouses humides et moyennement humides, Maghrébin*.

Oukaimeden, Tichka.

Paronychia polygonifolia (Vill.) DC.

Pelouses moyennement humides et sèches,

Méditerranéen*.

Ait Mizane, Oukaimeden, Tichka.

Sagina procumbens L.

Pelouses humides, bords des suintements, Cosmopolite*.

Ait Mizane, Tichka

Sagina saginoides subsp. parviflora Litard. \& Maire

I, R, Pozzines, suintements et pelouses très humides.

Ait Mizane, Oukaimeden, Tichka.

Silene heterodonta F.N.Williams

E, Pelouses moyennement humides et sèches.
Ait Mizane, Oukaimeden, Tichka.

Spergularia microsperma (Kindb.) Vved. subsp. oreophila (Litard \& Maire)

E, R, Pozzines.

Ait Mizane.

\section{CISTACEAE}

Helianthemum croceum (Desf.) Pers. subsp. suffruticosum (Boiss)

I, RR, Pelouses moyennement humides,

Méditerranéen.

Oukaimeden, Tichka.

\section{CRASSULACEAE}

Crassula vaillantii (Willd.) Roth

Zone inondable aux bords du lac, S et C

Europe, Syrie, C Asie, dés Maroc à Lybie, Ethiopie, S Afrique**.

Oukaimeden.

Sedum melanantherum DC.

I, R, Pozzines.

Ait Mizane, Tichka.

Sedum surculosum Coss.

E, suintements et pelouses très humides.

Ait Mizane, Oukaimeden, Tichka.

\section{DIPSACACEAE}

\section{Scabiosa atropurpurea $\mathrm{L}$.}

Pelouses humides et moyennement humides,

Région Méditerranéenne et Macaronésienne**. Oukaimeden, Tichka.

\section{GENTIANACEAE}

Blakstonia grandifolia (Viv.) Pau Pelouses humides, SW Région Méditerranéenne**. Oukaimeden, Tichka.

Centaurium erythrea Rafn subsp. suffruticosum (Griseb.) Greuter

Pelouses humides, SW Région

Méditerranéenne**.

Oukaimeden, Tichka.

Gentiana atlantica Litard. \& Maire

E, R, Pozzines et pelouses très humides.

Ait Mizane, Oukaimeden, Tichka.

Gentiana tornezyana Litard. \& Maire

E, Pozzines

Ait Mizane. 


\section{GERANIACEAE}

\section{Erodium cicutarium L.}

Pelouses moyennement humides et sèches, Eurasiatique-Méditerranéen*.

Ait Mizane, Oukaimeden, Tichka.

\section{Geranium cinereum Cav.}

E, Pozzines.

Ait Mizane

Geranium pyrenaicum Burm.f.

Pelouses humides, Européen-Méditerranéen*.

Ait Mizane, Oukaimeden.

\section{HYPERICACEAE}

\section{Hypericum pubescens Boiss.}

Pelouses humides, Ouest-méditerranéen**.

Oukaimeden.

\section{LABIATAE}

Hyssopus officinalis subsp. pilifer (Pant.) Murb.

$\mathrm{R}$ ?, pelouses moyennement humides, Eurasiatique***. Tichka.

Mentha suaveolens Ehrh. subsp. timija (Briq.)

$\mathrm{E}, \mathrm{R}$, pelouses humides.

Ait Mizane, Oukaimeden, Tichka.

Nepeta stachyoides Batt.

E, pelouses moyennement humides.

Ait Mizane, Oukaimeden.

Stachys arenaria Vahl. subsp. divaricatidens H.

Lindb.fil

$\mathrm{E}$, pelouses moyennement humides.

Oukaimeden, Tichka.

Thymus atlanticus (Ball) Roussine humides.

E, pelouses humides et moyennement

Ait Mizane, Oukaimeden, Tichka.

\section{LEGUMINOSEAE}

\section{Cicer atlanticum Maire}

E, RR, Pelouses humides et moyennement humides.

Tichka.

Lathyrus pratensis L.

Pelouses moyennement humides, Europe, Asie, N Afrique**.

Tichka.

\section{Lotus corniculatus L.}

Pelouses très humides, humides et moyennement humides, Eurasiatique-méditerranéen*.

Ait Mizane, Oukaimeden, Tichka.

Trifolium campestre Schreb.

Pelouses humides et moyennement humides,

Europe et Région méditerranéenne**.

Oukaimeden, Tichka.

Trifolium dubium Sibth.

Pelouses humides, Europe, NW Afrique, Région Macaronésienne**.

Oukaimeden, Tichka.

Trifolium humile L.

E, Pozzines, bords des ruisseaux et pelouses humides.

Ait Mizane, Oukaimeden, Tichka.

Trifolium ochroleucon Huds.

Pelouses moyennement humides, W et $\mathrm{C}$ Europe et Région méditerranéenne**.

Oukaimeden.

Trifolium repens $\mathrm{L}$.

Pelouses humides et bords des ruisseaux, Eurasiatique-méditerranéen*.

Ait Mizane, Oukaimeden, Tichka.

Trifolium strictum $\mathrm{L}$.

Pelouses humides, W Europe, Région méditerranéenne**.

Oukaimeden, Tichka.

\section{MALVACEAE}

\section{Malva tournefortiana $\mathrm{L}$.}

Pelouses moyennement humides et sèches, SW Europe, NW Afrique**.

Oukaimeden, Tichka.

\section{ONAGRACEAE}

Epilobium atlanticum Litard. \& Maire

I, RR, Pozzines et ruisselets.

Ait Mizane.

Epilobium obscurum Schreber.

RR, Pelouses humides, sources et ruisselets, Européen*****.

Oukaimeden, Tichka.

\section{OROBANCHACEAE}

Orobanche purpurea Jacq. 
R?, Pelouses humides. Eurasiatique***.

Tichka.

\section{PLANTAGINACEAE}

\section{Plantago coronopus L.}

Pelouses humides et moyennement humides, Eurasiatique-méditerranéen*.

Ait Mizane, Oukaimeden, Tichka.

\section{PLUMBAGINACEAE}

\section{Armeria atlantica Pomel}

Pelouses moyennement humides, Maroc, Algérie, Tunisie**.

Oukaimeden, Tichka.

\section{POLYGONACEAE}

Persicaria bistrota L. subsp. bistrota

RR, Pozzines et pelouses humides, Boréomontagnard*.

Oukaimeden, Tichka.

\section{Rumex acetosa $\mathrm{L}$.}

Suintements et Pelouses humides, Subcosmopolite**.

Oukaimeden, Tichka.

Rumex atlanticus Batt.

E, R, Pozzines et pelouses humides.

Ait Mizane, Oukaimeden, Tichka.

Rumex induratus Boiss. \& Reuter

Pelouses humides, Ibéro-maghrébin*.

Tichka

\section{PORTULACACEAE}

Montia fontana L. subsp. amporitana Sennen, Suintements. W et SW Europe, NW Afrique**. Oukaimeden.

\section{PRIMULACEAE}

\section{Anagallis tenella $\mathrm{L}$.}

Pelouses très humides et suintantes, Régions tempérées de l'hémisphère Nord**.

Oukaimeden, Tichka.

\section{RANUNCULACEAE}

Delphinium balansae Boiss. \& Reut.
Oukaimeden, Tichka.

Ranunculus aurasiacus Pomel

A, R, Pelouses humides.

Ait Mizane, Oukaimeden, Tichka.

Ranunculus bulbosus L. subsp. bulbosus

$\mathrm{R}$ ?, Pelouses humides et bords des ruisseaux, Eurosibérien****.

Oukaimeden, Tichka.

Ranunculus dyris (Maire) H. Lindb.f

E, R, Pelouses humides et bords des ruisseaux.

Ait Mizane, Oukaimeden, Tichka.

Ranunculus lateriflorus DC.

R ?, Bords du lac, Européen-méditerranéen*.

Oukaimeden.

Ranunculus paludosus subsp. leucothrix (Ball.)

Pelouses moyennement humides, S Europe, N Afrique, W Asie**.

Oukaimeden, Tichka.

\section{ROSACEAE}

Alchemilla atlantica Lindb.

$\mathrm{E}, \mathrm{RR}$, Pozzines et pelouses très humides.

Oukaimeden.

Aphanes cornucopioides Lag.

I, R, Pelouses moyennement humides.

Ait Mizane, Oukaimeden.

Potentilla erecta (L.) Hampe

R?, Bords des ruisseaux, Europe, Caucase,

Anatolie, Maroc, N Asie et N Amérique**.

Oukaimeden, Tichka.

Potentilla pennsylvanica L. subsp. hispanica (Zimmeter) Maire

R?, Pelouses moyennement humides, Ouestméditerranéen*.

Ait Mizane, Oukaimeden, Tichka.

Potentilla tornezyana Maire

E, R, Pozzines et pelouses humides

Ait Mizane, Tichka.

\section{RUBIACEAE}

\section{Galium parisiense L.}

Pelouses moyennement humides, $\mathrm{S}, \mathrm{W}$ et $\mathrm{C}$ Europe, E Hungarie et Bulgarie, Macaronésie, N Afrique, SW Asie**.

Tichka.

SAXIFRAGACEAE

Parnassia palustris L. 
Pozzines, Boréo-montagnard*.

Ait Mizane, Oukaimeden, Tichka.

Saxifraga granulata L.

Pelouses humides, Europe, W Asien NW Afrique**.

Ait Mizane, Oukaimeden, Tichka.

\section{SCROPHULARIACEAE}

Euphrasia willkomii Freyn.

I, RR, Pozzines.

Ait Mizane, Oukaimeden, Tichka.

Linaria micrantha (Cav.) Hoffman \& Link

Pelouses moyennement humides,

Méditerranéen**.

Oukaimeden, Tichka.

Linaria multicaulis L. subsp. pseudosupina (Ball)

Dobignad

E, Pelouses moyennement humides.

Oukaimeden, Tichka.

Parentucellia viscosa (L). Caruel

Pelouses très humides et suintements, $\mathrm{W}$ et $\mathrm{S}$

Europe, N Afrique, SW Asie, Macaronésie**.

Oukaimeden.

Veronica anagallis aquatica $\mathrm{L}$.

Bords des ruisseaux et pelouses très humides,

Eurasiatique-méditerranéen*.

Oukaimeden, Tichka.

Veronica beccabunga $\mathrm{L}$.

Bords des ruisseaux, Eurasiatique*.

Oukaimeden, Tichka.

Veronica repens $\mathrm{DC}$.

R?, Pozzines et bords des ruisseaux, Ouestméditerranéen*.

Ait Mizane, Oukaimeden, Tichka.

Veronica rosea Desf.

IA, Pelouses moyennement humides.

Ait Mizane, Oukaimeden, Tichka.

\section{THYMELACEAE}

\section{Daphne laureola $\mathrm{L}$.}

Pelouses très humides suintantes et ombragées.

Européen-méditerranéen*.

Tichka.

\section{VIOLACEAE}

Viola dyris Maire

$\mathrm{E}, \mathrm{R}$ ?, pelouses moyennement humides et sèches. Tichka.
Viola palustris L.

R?, Pozzines et pelouses très humides, Boréomontagnard*.

Ait Mizane, Oukaimeden, Tichka.

\section{ANGIOSPERMES MONOCOTYLEDONES AMARYLLIDACEAE}

Colchicum lusitanicum Brot.

Pelouses moyennement humides et sèches, Péninsule Ibérique, Italie, $\mathrm{N}$ Afrique**.

Oukaimeden.

Narcissus bulbocodium L. subsp. obesus (Salisb.)

Maire

I, RR, Pozzines.

Ait Mizane, Oukaimeden, Tichka.

\section{CYPERACEAE}

Carex depressa subsp. depressa Link.

R, Pozzines, Ouest-méditerranéen**.

Ait Mizane.

Carex distans L. subsp. distans

Pelouses humides, Europe, Macaronésie, N Afrique, W Asie**.

Oukaimeden, Tichka.

Carex divisa Huds.

Pelouses très humides et humides, Eurasiatiqueméditerranéen*.

Oukaimeden, Tichka.

Carex echinata Mur.

$\mathrm{R}$, Pozzines et pelouses très humides, Europe, Macaronésie, W et E Asie, N Amérique, Australie, Nouvelle Zelande**.

Oukaimeden, Tichka.

Carex flava $\mathrm{L}$.

$\mathrm{R}$, Pelouses très humides et suintements, Europe, N Amérique**.

Oukaimeden, Tichka.

Carex intricata Tineo

Pozzines, Supra-Méditerranéen*****.

Ait Mizane, Oukaimeden, Tichka.

Carex leporina $\mathrm{L}$.

R, Pozzines et suintements, Circumboréal $* * *$.

Ait Mizane, Oukaimeden, Tichka.

Eleocharis palustris L. Roem. \& Schult.

Suintements, bords du lac, Subcosmopolite**. 
Oukaimeden.

Eleocharis quinqueflora (Hartm.) O. Schwartz

Suintements, bords du lac, $\mathrm{N}$ et Europe montagneuse, Sibérie Caucase, Himalaya, N Amérique**.

Oukaimeden.

\section{HYACINTHACEAE}

Gagea dyris Maire

Pozzines, S. Espagne, Maroc, Algérie**.

Ait Mizane.

\section{Ornithogalum umbellatum $\mathrm{L}$.}

Pelouses humides et moyennement humides, Européen-méditerranéen*.

Ait Mizane, Oukaimeden, Tichka.

Urginea maritima (L.) Baker var. maura (Maire)

Pelouses moyennement humides, S Europe, Macaronésie, SW Asie, N Afrique**.

Oukaimeden, Tichka.

\section{IRIDACEAE}

\section{Crocus albiflorus Kit}

Pelouses suintantes, humides, moyennement humides et sèches, Subcosmopolite*****.

Oukaimeden, Tichka.

Gladiolus communis L. subsp. byzanthinus (Mill.)

A.P.Hamilton

Pelouses humides et moyennement humides, S Espagne, Sicile, NW Afrique**.

Oukaimeden, Tichka.

\section{JUNCACEAE}

Juncus bufonius L. subsp. mogadorensis H. Lindb.

Fil

$\mathrm{E}, \mathrm{RR}$, pelouses humides et bords des ruisseaux et du lac.

Oukaimeden, Tichka.

Juncus fontanesii Gay subsp. brachyanthus Trab. Pozzines, pelouses très humides. Région méditerranéenne, E Afrique et $\mathrm{W}$ Asie**.

Ait Mizane, Oukaimeden, Tichka.

Juncus inflexus L.

Pelouses humides et bords des ruisseaux, Europe, N, S, et E Afrique, $\mathrm{W}$ et C Asie**.

Oukaimeden, Tichka.

Luzula multiflora (Retz) subsp. congesta (Thuill.)
Hyl.

RR, Pozzines, Europe méridionale, centrale et occidentale ${ }^{* * * * *}$

Oukaimeden.

Luzula spicata (L.) DC.

RR, Pozzines, Europe, Asie, Amérique boréale ${ }^{* * * * *}$.

Oukaimeden, Tichka.

\section{ORCHIDACEAE}

Dactylorhiza elata (Poir.)

Pozzines, S Espagne, N Afrique**.

Oukaimeden, Tichka.

Orchis coriophora L.

Pozzines et Pelouses suintantes, S Espagne, N Afrique**.

Oukaimeden, Tichka.

Orchis mascula Link.

Pozzines et pelouses suintantes, W Europe, région méditerranéenne et Canaries**.

Oukaimeden, Tichka.

\section{POACEAE}

Agrostis atlantica Maire \& Trab.

E, R, Pozzines et pelouses humides.

Ait Mizane, Oukaimeden, Tichka.

Alopecurus pratensis L.

Pelouses humides, Bords des ruisseaux et du lac, Européen-méditerranéen*.

Oukaimeden, Tichka.

Anthoxanthum odoratum L.

Pozzines, pelouses humides et moyennement humides, Eurasiatique-méditerranéen*.

Ait Mizane, Oukaimeden, Tichka.

Arrhenatherum elatius L. subsp. bulbosum

(Wild.)

Pelouses moyennement humides et sèches prés des Azib, (rudérale), Europe, W Asie et NW Afrique**.

Oukaimeden, Tichka.

Avenula bromoides (Gouan) H.Scholz

Pozzines et pelouses humides, SW Europe et N Afrique**.

Ait Mizane Oukaimeden, Tichka.

Briza media L. subsp. media

R, Pozzines et pelouses humides, Eurasiatique****.

Oukaimeden, Tichka.

Bromus hordeaceus L. 
Pelouses humides et moyennement humides, Européen-méditerranéen*.

Oukaimeden, Tichka.

\section{Bromus squarrosus L.}

Pelouses humides et moyennement humides, Eurasiatique-méditerranéen*.

Oukaimeden, Tichka.

\section{Bromus tectorum L.}

Pelouses humides et moyennement humides, Eurasiatique-méditerranéen*.

Ait Mizane, Oukaimeden, Tichka.

Dactylis glomerata L. subsp. hispanica (Roth)

Nyman

Pelouses humides et moyennement humides,

Eurasiatique-méditerranéen*.

Oukaimeden, Tichka.

Deschampsia caespitosa (L.) P.B.

Pozzines, pelouses très humides et humides, Boréo-montagnard*.

Oukaimeden, Tichka.

Festuca atlantica duval-jouve subsp. oxyphylla

(Litard. \& Maire)

E, R, pelouses humides et moyennement humides.

Oukaimeden, Tichka.

Festuca maroccana Batt. \& Trabut. subsp. pozzicola

(Litard. \& Maire)

E, RR, pelouses moyennement humides.

Ait Mizane, Oukaimeden, Tichka.

Festuca mairei St Yves

Pelouses moyennement humides et sèches, Maroc, Algérie, Tunisie.

Oukaimeden.

Festuca rubra L.

Pozzines, pelouses très humides et humides, Holarctique $^{* * * *}$.

Ait Mizane, Oukaimeden, Tichka.

Festuca yvesii Litard.

E, Pelouses humides et moyennement humides.

Ait Mizane, Tichka.

Haynaldia ordacea Coss. \& DR.

Pelouses humides, Maroc et Algérie**.

Oukaimeden, Tichka.

Holcus lanatus L.

Pelouses très humides, humides et moyennement humides, Régions tempérées de l'hémisphère Nord**.

Oukaimeden, Tichka.

Koeleria crassipes Lange
Pelouses très humides et humides, Péninsule

Ibérique et NW Afrique**.

Oukaimeden, Tichka.

Lolium multiflorum Lam.

Pelouses très humides et humides, $\mathrm{W}$ et $\mathrm{C}$ de l'Europe, Région méditerranéenne et SW Asie**.

Oukaimeden, Tichka.

Nardus stricta L.

R, Pozzines, Boréo-montagnard*.

Ait Mizane, Oukaimeden, Tichka.

Phleum bertolonii DC.

Pelouses humides et moyennement humides, Europe, Région Irano-turaniènne et NW Afrique**.

Tichka.

Phleum pratens $\mathrm{L}$.

Pozzines et Pelouses humides, Circumboréal $* * *$.

Oukaimeden, Tichka.

Poa alpina L. subsp. atlantica (Trabut.)

E, R?, Pozzines.

Ait Mizane, Oukaimeden, Tichka.

Poa bulbosa L. var. vivipara Koel.

Pozzines, pelouses très humides et humides,

Europe, NW Afrique et Asie**.

Ait Mizane, Oukaimeden, Tichka.

Poa pratensis L. subsp. pratensis

$\mathrm{RR}$, Bords des ruisseaux, pelouses très humides et humides, Circum-boréal ${ }^{* * *}$.

Oukaimeden, Tichka.

Poa trivialis L.

Pozzines, pelouses très humides et humides, Europe, Asie, N Afrique, Macaronésie**.

Ait Mizane, Oukaimeden, Tichka.

Trisetum flavescens (L.) Beauv.

Pelouses humides et moyennement humides, Hémisphère Nord**.

Oukaimeden.

Vulpia geniculata (L.) Link subsp. pauana (Font Quer) Maire

E, RR, Pelouses humides et moyennement humides.

Oukaimeden, Tichka.

\section{DISCUSSION ET CONCLUSION}

La flore des pelouses humides dans les trois sites renferme une richesse spécifique estimée à 165 taxons répartis en 117 genres 
et 37 familles. Cette richesse floristique représente $13,6 \%$ de la richesse spécifique globale de la flore du Haut Atlas. La famille des Poaceae est la plus représentée (17\%) suivie de la famille des Asteraceae (10,2\%) et c'est le cas pour les trois sites dont les deux familles sont représentées respectivement par $16 \%$ et $13 \%$ à Ait Mizane, $20 \%$ et $10,4 \%$ à l'Oukaimeden et $19,4 \%$ et $10 \%$ à Tichka.

Les hémicryptophytes sont les plus représentés (60\%). Quézel (1957) a décrit les pozzines comme étant formées essentiellement d'hémicryptophytes, cette abondance reflète le caractère semi permanent de l'immersion des pelouses humides favorable à la prolifération d'espèces hygrophiles (Chevassut, 1956 in Hammada, 2007).

L'origine biogéographique ou encore la chorologie est l'un des principaux paramètres qui reflètent l'originalité de la flore des pelouses humides d'altitude. Plusieurs travaux portent sur la biogéographie de la flore Nordafricaine entre autres (Quézel, 1957, 1995, 2000; Pignatti, 1978; Galàn de Mera et al., 2003). Pour cette étude nous nous sommes basés sur le travail de Galland (1988) qui porte sur la caryologie et la cytogéographie de la flore orophile marocaine et sa relation avec l'origine et l'évolution de cette flore, complété par d'autres références en particulier des flores qui contiennent des listes de plantes avec leurs répartitions biogéographiques (Quézel et Santa 1962-1963; Galland, 1988; Mateo Sanz \& Crespo, 2001; Valdés et al., 2002).

Bien qu'on ne retrouve pas ces mêmes catégories citées par Galland (1988) dans les autres références utilisées nous nous sommes intéressés dans notre étude aux taxons nordiques caractéristiques des grands complexes continentaux septentrionaux (Quézel, 2000) et dont la présence dans les montagnes Nord-africaines et spécialement dans les pozzines constituent l'originalité de ces milieux (Quézel, 1957; Galland, 1988).

Ces taxons de souches septentrionales représentent $15,7 \%$ de l'ensemble des taxons répertoriés dans les pelouses humides dans les trois sites étudiés. Ces taxons d'origine biogéographique remarquable occupent essentiellement les pozzines et le faciès très humides. Ils représentent $11,6 \%$ dans la partie amont de la haute vallée d'Ait Mizane, $18 \%$ des taxons inventoriés sur les pelouses humides du plateau de Tichka et $17,4 \%$ sur le plateau de l'Oukaimeden (tab. 1).

Dans les pelouses humides de haute montagne en l'occurrence au niveau des sites d'Ait Mizane, d'Oukaimeden et de Tichka le taux d'endémisme global atteint 30,3\%. Ce taux dépasse celui des zones humides du Maroc (ne contenant pas les zones humides de montagne) qui est de 10\% (Hammada, 2007) et celui du Haut Atlas qui est de 25,8\% (Ouhammou, 2005). Sur les 165 espèces inventoriées dans nos sites d'étude, 41 espèces sont endémiques stricts du Maroc soit 24,8\% (tab. 2).

Bien que le taux d'endémisme global soit assez important il diffère néanmoins d'un site à l'autre, et loin d'être proportionnel à la richesse spécifique. Par ailleurs, Ouhammou 2003 et 2005 a bien montré la forte corrélation entre l'altitude et le taux d'endémisme.

Ait Mizane est le site le plus riche en espèces endémiques. Le taux d'endémisme est très important et dépasse $50 \%$ suivi du plateau du Tichka qui comprend un taux d'endémisme global de $30,2 \%$ et puis le plateau d'Oukaimeden où le taux d'endémisme est de $27 \%$.

Le degré de rareté dans l'ensemble des taxons répertoriés dans nos sites d'études est estimé à 31\%. Sur les 165 taxons, 21 espèces sont rares, 17 sont très rares, 12 sont soupçonnées rares et 1 espèce est soupçonnée très rare, nous n'avons pas noté la présence de taxons éteints ou vulnérables. Le degré de rareté sur les pelouses humides de l'amont de la vallée d'Ait Mizane est estimé à 40,5\%, Sur le plateau de Tichka il est de $31 \%$ et sur le plateau de l'Oukaimeden 


\begin{tabular}{|c|c|c|c|}
\hline Les taxons de souches septentrionales & Catégories chorologiques & Faciès de pelouses humides & Localités \\
\hline \multirow[t]{2}{*}{ Cerastium arvense $\mathrm{L}$. } & Boréo-montagnard & Pozzines & Ait Mizane, Oukaimeden, \\
\hline & & & Tichka \\
\hline \multirow[t]{2}{*}{ Cerastium cerastoides $\mathrm{L}$. } & Boréo-montagnard & Pozzines & Ait Mizane, Oukaimeden, \\
\hline & & & Tichka \\
\hline Deschampsia caespitose $\mathrm{L}$. & Boréo-montagnard & Pozzines, pelouses très humides et humides & Oukaimeden, Tichka \\
\hline \multirow[t]{2}{*}{ Nardus stricta $\mathrm{L}$. } & Boréo-montagnard & Pozzines & Ait Mizane, Oukaimeden, \\
\hline & & & Tichka \\
\hline \multirow[t]{2}{*}{ Parnassia palustris L. } & Boréo-montagnard & Pozzines & Ait Mizane, Oukaimeden, \\
\hline & & & Tichka \\
\hline Persicaria bistrota $\mathrm{L}$. & Boréo-montagnard & Pozzines et pelouses humides & Oukaimeden, Tichka \\
\hline \multirow[t]{2}{*}{ Viola palustris $\mathrm{L}$. } & Boréo-montagnard & Pozzines et pelouses très humides & Ait Mizane, Oukaimeden, \\
\hline & & & Tichka \\
\hline \multirow[t]{2}{*}{ Asplenium trichomanes $\mathrm{L}$. } & Circum-boréal & Suintements et pelouses & Tichka \\
\hline & & très humides & \\
\hline \multirow[t]{2}{*}{ Carex leporina $\mathrm{L}$. } & Circum-boréal & Pozzines et suintements & Ait Mizane, Oukaimeden, \\
\hline & & & Tichka \\
\hline Phleum pratens $\mathrm{L}$ & Circum-boréal & Pozzines et Pelouses humides & Oukaimeden, Tichka \\
\hline \multirow[t]{2}{*}{ Poa pratensis $\mathrm{L}$. } & Circum-boréal & Bords des ruisseaux, pelouses très humides & Oukaimeden, Tichka \\
\hline & & et humides & \\
\hline Briza media L. Ssp. Media & Eurasiatique & Pozzines et pelouses humides & Oukaimeden, Tichka \\
\hline Hyssopus officinalis Punt. & Eurasiatique & Pelouses moyennement humides & Tichka \\
\hline Orobanche pupurea Jaq. & Eurasiatique & Pelouses humides & Tichka \\
\hline Veronica Beccabunga $\mathrm{L}$. & Eurasiatique & Bords des ruisseaux & Oukaimeden, Tichka \\
\hline Epilobium obscurum Schreber. & Européen & Pelouses humides, sources et ruisselets & Oukaimeden, Tichka \\
\hline Ranunculus bulbosus Willk. & Eurosibérien & Pelouses humides et bords des ruisseaux & Oukaimeden, Tichka \\
\hline Trisetum flavescens L. & Hémisphère Nord & Pelouses humides et moyennement humides & Oukaimeden \\
\hline \multirow[t]{2}{*}{ Festuca rubra $\mathrm{L}$. } & Holarctique & Pozzines, pelouses très humides et humides & Ait Mizane, Oukaimeden, \\
\hline & & & Tichka \\
\hline Conium maculatum $\mathrm{L}$. & Nord Afrique, Eurasie & Pelouses humides & Oukaimeden, Tichka \\
\hline \multirow[t]{2}{*}{ Anagallis tenella $\mathrm{L}$. } & Régions tempérées de l'hémisphère & Pelouses très humides et suintantes & Oukaimeden, Tichka \\
\hline & Nord & & \\
\hline \multirow[t]{2}{*}{ Holcus lanatus L. } & Régions tempérées de l'hémisphère & Pelouses très humides, humides et & Oukaimeden, Tichka \\
\hline & Nord & moyennement humides & \\
\hline \multirow[t]{2}{*}{ Botrychium lunaria L. } & Europe, Asie tempérée, N Amérique, & Pozzines, suintements et pelouses très & Ait Mizane, Oukaimeden, \\
\hline & Patagonie, Australie, Tasmanie & humides & Tichka \\
\hline \multirow[t]{2}{*}{ Carex echinata Mur } & Europe, Macaronésie, W et $\mathrm{E}$ Asie, $\mathrm{N}$ & Pozzines et pelouses très humides & Oukaimeden, Tichka \\
\hline & Amérique, Australie, Nouvelle Zelande & & \\
\hline Luzula spicata (L.) DC & Europe, Asie, Amérique boréale & Pozzines & Oukaimeden, Tichka \\
\hline \multirow[t]{2}{*}{ Potentilla erecta $\mathrm{L}$. } & Europe, Caucase, Anatolie, Maroc, N & Bords des ruisseaux & Oukaimeden, Tichka \\
\hline & Asie et N Amérique & & \\
\hline
\end{tabular}

Tableau 1: Liste des taxons de souches septentrionales recensés dans nos sites d'études selon les catégories chorologiques. List of northern taxa recorded in the studied sites. 
le degré de rareté est de 29\%. Les pozzines constituent ainsi d'excellents refuges pour les taxons rares (Fennane \& Ibn Tattou, 1994)

L'analyse floristique des pelouses humides dans la zone d'étude (le plateau de l'Oukaimeden, l'amont de la haute vallée d'Ait Mizane et le plateau de Tichka) a permis de déceler que ces sites étudiés représentent un important centre de phytobiodiversité « hot spot » (Médail \& Quézel, 1997; Médail, 2001). Ce centre est lié à l'histoire et aux conditions écologiques particulières passées et actuelles qui ont favorisé localement le développement d'une flore riche et diversifiée et souvent endémique.

Cependant, cette biodiversité floristique se trouve actuellement menacée du moment que le degré de rareté global dans les trois sites étudiées est de $31 \%$, dont $23 \%$ sont des taxons rares et très rares. Cette vulnérabilité est encore plus marquante, surtout que $39,4 \%$ des taxons rares et très rares sont des endémiques stricts du Maroc et 25,6\% sont de souches septentrionales. Cette flore rare et son importance reflètent la vulnérabilité de cette végétation et la fragilité de ses habitats.

Il est à signaler que sur la totalité de la flore des pelouses humides des trois sites, les taxons de souches septentrionales comportent un degré de rareté de $61 \%$ d'où leur vulnérabilité et la nécessité de leur protection pour protéger l'histoire botanique de tout le Haut Atlas.

De par leur déterminisme hydrique ces pâturages humides sont très touchées par les changements climatiques (Hulme, 2005; Sebastià, 2007). Ces milieux sont affectés au niveau de leur structure, et fonctionnement écologique. L'étude diachronique, réalisée sur les pelouses humides du plateau d'Oukaimeden (entre 1986 et 2004), a montré que $8 \%(6,598$ ha) de ces milieux est en régression (Alaoui Haroni et al., 2009b). Par leur sensibilité au climat et au facteur hydrique, les pozzines et les pelouses humides fragilisées d'avantage par les changements climatiques subissent une pression humaine de plus en plus croissante; urbanisation, activités touristiques, pollution... on assiste ainsi à la régression d'un grand foyer de la biodiversité de la flore orophile du Haut Atlas. Cependant et heureusement, le mode de gestion Agdal encore maintenu dans la majorité des parcours du Haut Atlas (Oukaimeden et Tichka) contribue efficacement à la préservation de la biodiversité dans ces sites. La régression atteint la superficie de ces espaces et non pas leur composition. Les Agdals pastoraux apparaissent donc comme des aires

\begin{tabular}{cccc}
\hline & $\begin{array}{c}\text { Ait Mizane } \\
(69 \text { taxons })\end{array}$ & $\begin{array}{c}\text { Tichka } \\
(140 \text { taxons })\end{array}$ & $\begin{array}{c}\text { Oukaimeden } \\
(134 \text { taxons })\end{array}$ \\
\hline E & 28 & 35 & 29 \\
I & 6 & 5 & 5 \\
A & 1 & 1 & 1 \\
IA & 1 & 1 & 1 \\
\hline
\end{tabular}

Tableau 2: Le nombre de taxons endémiques dans la zone d'étude. E: Endémique du Maroc; I: Endémique du Maroc et de la Péninsule Ibérique; A: Endémique du Maroc et de l'Algérie; IA: Endémique du Maroc, de la Péninsule Ibérique et de l'Algérie. The number of endemic taxa in the study area. E: Endemic of Morocco; I: Endemic of Morocco and Iberian Peninsula; A: Endemic of Morocco and Algeria; IA: Endemic of Morocco, Iberian peninsula and Algeria. 
protégées typiques garantissant la durabilité de la ressource et la gestion des conflits autour de cette ressource (Alifriqui, 2007).

La biodiversité donc est une notion qui ne peut être perçue en dehors des mutations sociales, humaines et gestionnaires que connaissent ces sites (Benabid, 2000; Waldhardt, 2003; O'rourke, 2006). Les modes de gestion et les types d'usage de ces milieux influeront fortement leur protection et préservation (Austrheim et al., 1999).

REMERCIEMENTS. Nos vifs remerciements au programme de recherche pluridisciplinaire «Les Agdal du Haut Atlas marocain: biodiversité et gestion communautaire de l'accès aux espaces sylvopastoraux » pour son appui financier et scientifique (Convention de financement IRDIFB / INRA $n^{\circ}$ 2886). Au projet de recherche «Conservation et valorisation des zones humides du SIBE Ait Mizane» mené par l'Association des Amis du Muséum d'Histoire Naturelle de Marrakech (AAMHNM). Nous présentons aussi notre grande gratitude au Professeur A. Ouhammou pour son aide précieuse pour la détermination des espèces.

\section{RÉFÉRENCES BIBLIOGRAPHIQUES}

ALAOUI HARONI, S. \& M. ALIFRIQUI 2009- When traditional management respects phenological cycles of plants in wet pastures in the Moroccan mountains. African Journal of Ecology (en cours).

ALAOUI HARONI, S., M. ALIFRIQUI \& V. SIMONNEAUX -2009- Recent Dynamic of the Wet Pastures at Oukaimeden Plateau (High Atlas mountains, Morroco). Biodiversity and conservation 18(1): 167-189.

ALIFRIQUI, M. -2007- Gérer la biodiversité dans le Haut Atlas. In Alifriqui M. \& Auclair L. (sous presse) «Les Agdals de l'Atlas marocain. Savoirs locaux, droits d'accès, gestion de la biodiversité » Edit. IRD/IRCAM.

AUCLAIR, L. \& M. ALIFRIQUI -2005- Les Agdals du Haut Atlas marocain. Enjeux d'une recherche pluridisciplinaire. Cahiers de recherche $d u$ Centre Jacques Berque, III, 61-79.

AUSTRHEIM, G., E. GUNILLA A. OBSON \&
E. GRONTVEDT -1999- Land-use impact on plant communities in semi - natural sub-alpine grassland of Budalen, central Norway. Biological Conservation 87: 369 - 379.

BENABID, A. -2000- Flore et écosystèmes du Maroc. Évaluation et préservation de la biodiversité. Ibis Press, Paris.

BERQUE, J. -1955- Structures sociales du Haut Atlas, Paris, P.U.F, $2^{\text {ème }}$ édition, 513 p.

DRESCH, J. -1941-Recherches sur l'évolution du relief dans le massif central du grand Atlas, le Haouz et le Sous. Tours, Arrault et Cie, Mautres imprimeries. 708p.

EL GRAOUI, M., M. ALIFRIQUI H. JUNGNER A. NAHID \& S.SEARIGHT-MARTINET -2008Recherche d'indice chronologique sur le passage des gravures de rochers à l'Oukaimeden (Haut Atlas, Maroc). Sahara 19: 105-108.

EMBERGER, L. \& MAIRE R. -1941- Catalogue des plantes du Maroc, volume IV. Minerva, Alger.

FENNANE, M. \& IBN TATTOU M. -1994- La flore rare des hautes montagnes marocaines. In Ges Rencontres de l'agence régionale pour l'environnement Provence-Alpes-Côte d'Azur.199-205.

FENNANE, M. \& IBN TATTOU M. -1998Catalogue des plantes vasculaires rares, menacées ou endémiques du Maroc. Bocconea, 8, 243p.

FENNANE, M., IBN TATTOU M., MATHEZ J., OUYAHYA A. \& EL OUALIDI J. -1999- Flore pratique du Maroc, 1. Trav. Inst. Sci. serv. Bot. $36,558 \mathrm{p}$.

FENNANE, M., IBN TATTOU M., OUYAHYAA. \& EL OUALIDI J. -2007- Flore pratique du Maroc, 2. Trav. Inst. Sci. serv. Bot. 38, 636p.

GALÁN DE MERA, A., A.V. PÉREZ-LATORRE \& J.A. VICENTE ORELLANA -2003- Relaciones fitogeográficas entre el suroccidente de la Península Ibérica y el noroeste de África. Una propuesta de sectorización. Lagascalia 23, 27-51.

GALLAND, N. -1988- Recherches sur l'origine de la flore orophile du Maroc. Etude caryologique et cytogèographique. Travaux de l'Inst. Sci., Série Botanique Rabat 35, 132p.

GAUQUELIN, T. -1988- Dynamique de la végétation et des formations superficielles dans les montagnes du bassin occidental de la méditerranée: étude des formations a genévrier thurifère et xérophytes épineuses en coussinet 
des atlas marocains; doctorat d'état; Université Paul Sabatier, Toulouse, Tome 1 :148p; Tome 2, Tab. \& fig.

GODRON, M. -1971- Comparaison d'une courbe aire-espèces et de son modèle. Oecol. Plant. 6, 189- 193.

GUINOCHET, M. -1973- La phytosociologie. Collection d'écologie I. Masson éd., Paris, $227 \mathrm{p}$.

HAMMADA, S. -2007- Etudes Sur La Végétation Des Zones Humides Du Maroc Catalogue et Analyse de la Biodiversité Floristique et Identification. Doctorat d'état ès-Sciences université Mohammed V -Agdal Rabat. 199p.

HULME, P E. -2005- Adapting to climate change: is there scope for ecological management in the face of a global threat? Journal of Applied Ecology 42(5): 784-794.

IBN TATTOU, M. \& M. FENNANE -1989- Aperçu historique et état actuel des connaissances sur la flore vasculaire du Maroc. Bull Inst Sci Rabat $13,85-94$.

ILAHIANE, H. -1990- The berber agdal institution: indigenous range management in the Atlas mountains. Ethnology 38: 21-45.

JAHANDIEZ, E. \& R. MAIRE -1931- Catalogues des plantes du Maroc. Tome premier: Ptéridophytes, Gymnospermes et Monocotylédones. Alger, Minerva, $50 \mathrm{p}$.

JAHANDIEZ, E. \& R. MAIRE -1932- Catalogue des plantes du Maroc. Tome deuxième: Dicotylédones Archichlamydées. Alger, Minerva, 151- 558.

JAHANDIEZ, E. \& R. MAIRE-1934- Catalogue des plantes du Maroc. Tome troisième: Dicotylédone Gamopétales et suppléments aux vol. I et II. Alger, Minerva, 559-913.

MAHDI, M. -1999- Pasteur de l'Atlas. Production pastorale, droit et rituel. Fond. Konrad Adenauer, (Ed.) Casablanca, 347p.

MATEO, SANZ G. \& M. B. CRESPO -2001Manual para la determinacion de la flora valenciana. Edit. Moliner-40. 503p.

MEDAIL, F. -2001- Les hot spots de biodiversite: un outil pour la conservation?: Comment conserver les espèces. Biofutur 211, 37.

MEDAIL, F. \& P. QUEZEL -1997- Hot-spot analysis for conservation of plant biodiversity in the Mediterranean basin. Ann. Missouri Bot. Gard. 84: 112-127.
O'ROURKE, E. -2006- Biodiversity and land use change on the Causse Me'jan, France. Biodiversity and Conservation 15: 2611-2626.

OUHAMMOU, A. -2003-Richesse spécifique et endémisme de la flore vasculaire dans la zone d'altitude du parc national de Toubkal, Haut-Atlas de Marrakech, Maroc. Naturalia Maroccana 1(1): 49-45.

OUHAMMOU, A. -2005-Flore et végétation du Parc National de Toubkal (Haut-Atlas de Marrakech, Maroc): typologie, écologie et conservation. Th. D'état. Univ. Cadi Ayyad, Fac. Sci. Marrakech, 260p.

PIGNATTI, S. -1978- Evolutionary trends in the Mediterranean flora and vegetation. Vegetatio 37: 175-185.

QUEZEL, P. -1957- Peuplement végétal des hautes montagnes de l'Afrique du nord. Encyclopédie biogéographique et écologique, édition $\mathrm{P}$. Lechvalier, Paris, $463 \mathrm{p}$.

QUEZEL, P. -1995- La flore du bassin méditerranéen: origine, mise en place, endémisme. Ecol. Medit., XXI, (12), 19-39.

QUEZEL, P. -2000- Réflexions sur l'évolution de la flore et de la végétation au Maghreb méditerranéen. Edit. Ibiss Press. 117p.

QUEZEL, P. \& S. SANTA -1962 - 1963- Nouvelle flore de l'Algérie et des régions désertiques méridionales. Editions C.N.R.S. Paris, Tomes I et II, $1165 \mathrm{p}$.

REILLE, M. -1976-Analyse pollinique de sédiments postglaciaires dans le moyen Atlas et le Haut Atlas marocains: Premiers résultats. Ecologia Mediterranea 2: 153-170.

SEBASTIÀ, M. T. -2007- Plant guilds drive biomass response to global warming and water availability in subalpine grassland. Journal of Applied Ecology 44(1): 158-167.

THINON, M. \& M. ALIFRIQUI -2004- Dégradation des thuriféraies marocaines: premières données phytohistoriques pédoanthracologiques. Naturalia Maroccana 2(1-2): 241-250.

VALDÉS, B., M. REJDALI, A. ACHHAL EL KADMIRI, S. L. JURY \& J. M. MONTSERRAT -2002- Catalogue des plantes vasculaires $d u$ Nord du Maroc, incluant des clés d'identification. Vol. I-II. (Ed.). 1007p.

WALDHARDT, R. -2003-Biodiversity and Landscapesummary, conclusions and perspectives. Agriculture, Ecosystems and Environment 98: 305-309. 